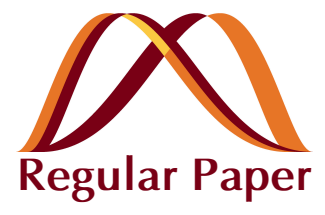

\title{
A Study of the Quantitative Relationship of Charge- Density Changes and the Design Area of a Fabricated Solar Cell
}

\author{
Kyeong-Nam Jeon \\ Department of Electronic and Optical Communications Engineering, Gwang-ju University, Gwangju 503-703, Korea
}

Seon-Hun Kim and Hoy-Jin Kim

Department of Research and Business, Korea Photonics Technical Institute, Gwangju 500-779, Korea

In-Sung Kim ${ }^{+}$and Sang-Hyun Kim

Department of Research, Semitech Corporation, Ahsan 336-708, Korea

Received March 26, 2011; Revised July 4, 2011; Accepted July 5, 2011

\begin{abstract}
In this paper, the design area of a fabricated solar cell has been analyzed with respect to its charge density. The mathematical calculation used for charge-density derivation was obtained from the 2001 version of a MATHCAD program. The parameter range for the calculations was $\pm 1 \times 10^{17} \mathrm{~cm}^{-3}$, which is in the normal parameter range for n-type doping impurities $\left(7.0 \times 10^{17} \mathrm{~cm}^{-3}\right)$ and also for $p$-type impurities $\left(4.0 \times 10^{17} \mathrm{~cm}^{-3}\right)$. Therefore, it can be said that the fabricated solar-cell design area has a direct effect on charge-density changes.
\end{abstract}

Keywords: Gallium arsenide, Silicon, Impurity, Doping, Fabricated design area, Charge density

\section{INTRODUCTION}

The determination of the fabricated design area in research and development of solar cells for high-efficiency power conversion is very important. There is a direct relationship between the design area and charge density in the $p-n$ junction depletion region. However, the scientific literature contains no direct studies of the effects on charge density of the design area of solar cells. The research described in this paper indicates how changes in the solar-cell design area can be used to affect charge density in fabrication design for both silicon and gallium arsenide (GaAs) solar cells. In addition, the thickness and the area of semicon-

${ }^{\dagger}$ Author to whom all correspondence should be addressed: E-mail: kis820926@nate.com

Copyright @2011 KIEEME. All rights reserved.

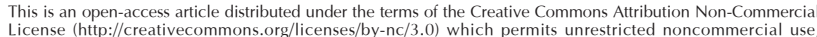
Llicense (http:///creativecommons.org/licenses/by-nc/3.0) which permits unrestricted
distribution, and reproduction in any medium, provided the original work is properly cited. ductor thin film in solar-cell fabrication was found to be related to the carrier lifetime. Therefore, the carrier lifetime of solar-cell semiconductor thin film is related to high-efficiency power conversion. Basically, the objective of increasing electron density in most solar-cell fabrication designs leads to a thicker design for negative types than for positive types.

Therefore, this design technology involves a set of technologies to increase power-conversion efficiency by increasing electron density. A typical device structure which is routinely used for high-efficiency device fabrication consists of a $p$ - $n$ silicon front contact and a GaAs material back contact, as shown in Fig. 1.

The following sequence of processing steps is generally used for device fabrication. This paper investigates the changes in charge density with various solar-cell fabrication design areas (width $[\mathrm{cm}] \times$ length $[\mathrm{cm}]$ ) for standard, type 1, type 2, type 3 , type 4 , and type 5 designs. In addition, the effects of chargedensity changes and current output were investigated as direct effects. The charge density is the total amount of charge in a fixed length or area divided by the material volume [1]. 


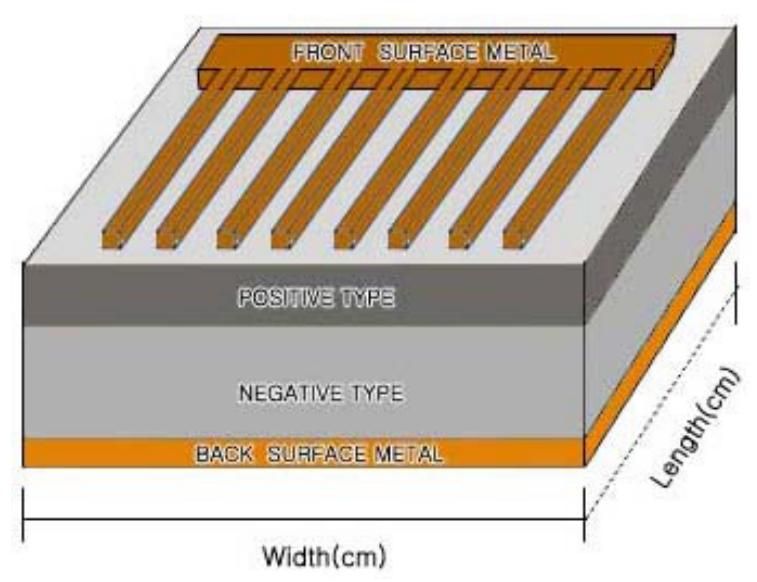

Fig. 1. Typical $p-n$ junction of solar-cell device structure.

\section{THEORETICAL BACKGROUND}

The electrical properties of semiconductors related to doping and to states which exist deep in the band gap can be conveniently measured using the depletion capacitance of a diode.

The present treatment of $p+n$ junctions will be limited, and it should be noted that the result for the depletion capacitance of Schottky diodes is identical. Both $p-n$ junctions and Schottky diodes can serve this purpose. However, minority carrier effects can be studied only with $p$ - $n$ junctions because Schottky diodes are majority carrier devices. The term "abrupt junctions" refers to junctions which contain ideally abrupt doping profiles as shown in Fig. 2(a), and the term "one-sided" means that the concentration of dopant is much higher on one side. Any variation lateral to the junction is ignored, and the overall junction must be neutral, which implies that $\mathrm{N}_{A} \mathrm{X}_{\mathrm{p}}=\mathrm{N}_{\mathrm{D}} \mathrm{X}_{\mathrm{n}}$. The solutions for the electric field and electrostatic potential are shown in Figs. 2(b) and (c). These can be straightforwardly obtained by integrating Poisson's equation, which for the $\mathrm{n}$ side is given by (meter-kilogram-second [MKS] units),

$$
-\frac{\partial^{2} V}{\partial x^{2}}=\frac{\partial E}{\partial x}=\frac{\rho(x)}{\varepsilon}=q \frac{N_{D}}{\varepsilon}
$$

where $\varepsilon$ is the dielectric constant and $q$ is the charge of an electron. The result for the electric field is:

$$
E(x)=\frac{q N_{D}}{\varepsilon}\left(x-x_{n}\right) \text { for } 0<x<x_{n}
$$

and that for the electrostatic potential is:

$$
V(x)=V_{c}-\frac{q N_{D}}{2 \varepsilon}\left(x_{n}-x\right)^{2}
$$

where $V_{x}$ is the integration constant corresponding to the total electrostatic potential across the diode. For a one-sided abrupt junction, $V(x=0) \cong 0$, which implies that:

$$
V_{c}=V_{b i}+V=\frac{q N_{D} x_{n}^{2}}{2 \varepsilon}
$$

(a)

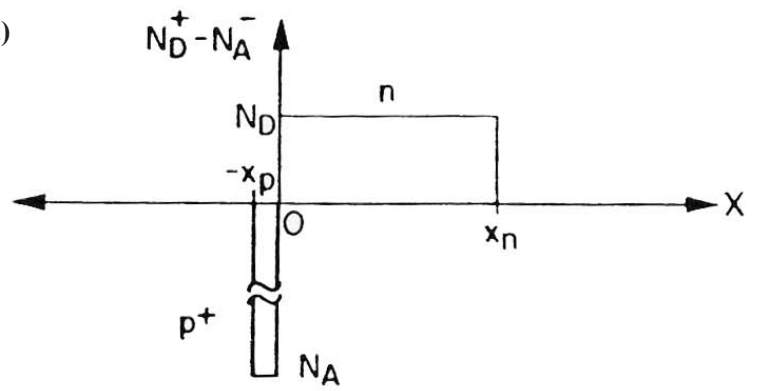

(b)

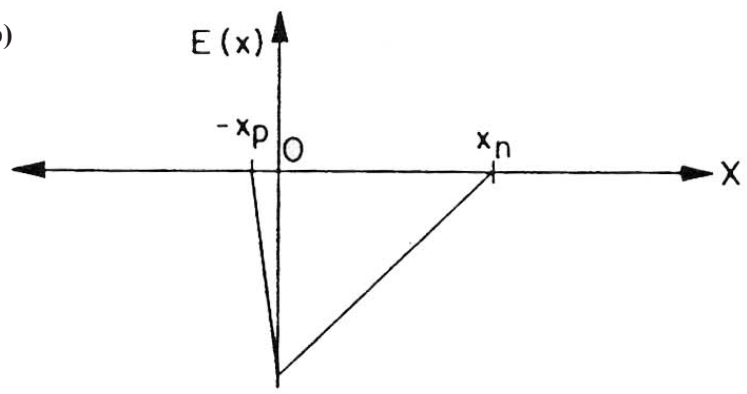

(c)

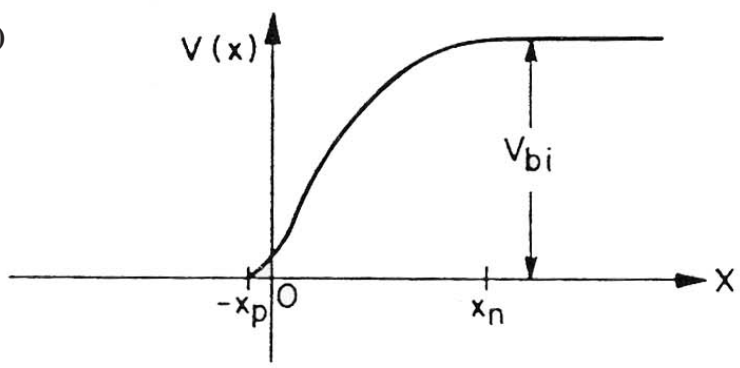

(d)

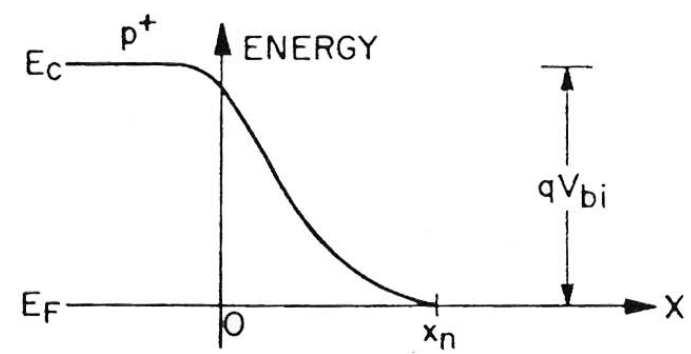

Fig. 2. (a) space charge, (b) electric field, (c) electrostatic potential, (d) electron energy for an abrupt $p+n$ junction diode.

where $V_{b i}$ is the "built-in" potential and $V$ is the applied bias. For one-sided and abrupt junctions, the depletion depth is given by:

$$
W=x_{n}+x_{p} \cong x_{n}
$$

The solution for the electrostatic potential is often drawn superimposed on the band structure as shown in Fig. 2(d) as a "band-bending diagram." Here, the Fermi level does not vary across the junction if no bias is applied because the Fermi level is the chemical potential of the carriers, which must be constant in equilibrium. The crossing point between the Fermi level and the donor level determines the depletion depth. If a slight bias, $\Delta V$, is applied, then according to Eq. $6, \Delta V=q N_{D} W \Delta W / \varepsilon$. Because the amount of donor charge which is uncovered at the edge of the depletion region is given by $\Delta Q=N_{D} A \Delta W$, where $A$ is the square of the diode, the differential capacitance is given by: 
Table 1. Concentration range of doping impurities for calculations.

\begin{tabular}{lc}
\hline Division & Concentration range of doping impurities \\
\hline n-type & $6.0 \times 10^{17} \mathrm{~cm}^{-3} \leq 7.0 \times 10^{17} \mathrm{~cm}^{-3} \leq 8.0 \times 10^{17} \mathrm{~cm}^{-3}$ \\
p-type & $3.0 \times 10^{17} \mathrm{~cm}^{-3} \leq 4.0 \times 10^{17} \mathrm{~cm}^{-3} \leq 5.0 \times 10^{17} \mathrm{~cm}^{-3}$ \\
\hline
\end{tabular}

Table 2. Experimental design for solar-cell charge-density analysis in the depletion region.

\begin{tabular}{|c|c|c|c|c|}
\hline \multicolumn{2}{|c|}{ Type } & Width $(\mathrm{cm})$ & Length $(\mathrm{cm})$ & Area $\left(\mathrm{cm}^{2}\right)$ \\
\hline \multirow[t]{2}{*}{ Standard } & 1 & 0.5 & 0.5 & 0.25 \\
\hline & 2 & 1 & 1 & 1 \\
\hline \multirow[t]{3}{*}{ Type 1} & 3 & 1.5 & 1.5 & 2.25 \\
\hline & 4 & 2 & 2 & 4 \\
\hline & 5 & 0.5 & 1 & 0.5 \\
\hline \multirow[t]{3}{*}{ Type 2} & 6 & 0.5 & 1.5 & 0.75 \\
\hline & 7 & 0.5 & 2 & 1 \\
\hline & 8 & 1 & 0.5 & 0.5 \\
\hline \multirow[t]{3}{*}{ Type 3} & 9 & 1 & 1.5 & 1.5 \\
\hline & 10 & 1 & 2 & 2 \\
\hline & 11 & 2 & 0.5 & 1 \\
\hline \multirow[t]{3}{*}{ Type 4} & 12 & 2 & 1 & 2 \\
\hline & 13 & 2 & 1.5 & 3 \\
\hline & 14 & 3 & 0.5 & 1.5 \\
\hline \multirow[t]{2}{*}{ Type 5} & 15 & 3 & 1 & 3 \\
\hline & 16 & 3 & 1.5 & 4.5 \\
\hline
\end{tabular}

$$
C=\frac{\Delta Q}{\Delta V}=\frac{\varepsilon A}{W}
$$

This is a basic result which is easy to remember because it is identical to that for a parallel-plate capacitor. If there is a quiescent applied bias, $V$, which increases the depletion depth (i.e., the reverse bias), then the capacitance is decreased, and this affords a convenient way to ascertain the polarity of a diode. It follows from Eqs. (4) and (6) that:

$$
\frac{A^{2}}{C^{2}}=\frac{W^{2}}{\varepsilon^{2}}=\frac{2}{q \varepsilon N_{D}}\left[V_{b i}+V\right]
$$

This key result implies that by plotting $A^{2} / C^{2}$ as a function of reverse bias, the space charge density can be obtained from the slope. A similar procedure can be shown to be valid even if the space charge density is not uniform. it is still true that:

$$
\frac{d}{d V}\left[\frac{A^{2}}{C^{2}}\right]=\frac{2}{q \varepsilon N_{D}(x)}
$$

which is often stated as:

$$
N(x)=-\frac{C^{3}}{q \varepsilon A^{2}}\left[\frac{d C}{d V}\right]^{-1}
$$

where $N(x)$ is the net space charge density in the depletion region. These relations are the basis for a popular doping profiler known as the "Miller profiler" which enables placement of a diode at the end of a coaxial cable of essentially arbitrary length. The charge distribution in the treatment was assumed to change abruptly from $N_{D}$ to zero at $x_{n}$. Actually, the electrons spill over

\begin{tabular}{|c|c|c|c|c|}
\hline \multicolumn{2}{|c|}{ Type } & \multirow{2}{*}{$\frac{\text { Width }(\mathrm{cm})}{0.5}$} & \multirow{2}{*}{$\begin{array}{c}\text { Length }(\mathrm{cm}) \\
0.5\end{array}$} & \multirow{2}{*}{$\begin{array}{c}\text { Multipliers } \\
1\end{array}$} \\
\hline Standard & 1 & & & \\
\hline & 2 & 1 & 1 & 4 \\
\hline \multirow[t]{2}{*}{ Type 1} & 3 & 1.5 & 1.5 & 9 \\
\hline & 4 & 2 & 2 & 16 \\
\hline \multirow[t]{4}{*}{ Type 2} & 5 & 0.5 & 1 & 2 \\
\hline & 6 & 0.5 & 1.5 & 3 \\
\hline & 7 & 0.5 & 2 & 4 \\
\hline & 8 & 1 & 0.5 & 2 \\
\hline \multirow[t]{3}{*}{ Type 3} & 9 & 1 & 1.5 & 6 \\
\hline & 10 & 1 & 2 & 8 \\
\hline & 11 & 2 & 0.5 & 4 \\
\hline \multirow[t]{2}{*}{ Type 4} & 12 & 2 & 1 & 8 \\
\hline & 13 & 2 & 1.5 & 12 \\
\hline \multirow[t]{3}{*}{ Type 5} & 14 & 3 & 0.5 & 6 \\
\hline & 15 & 3 & 1 & 12 \\
\hline & 16 & 3 & 1.5 & 18 \\
\hline
\end{tabular}
into the depletion region according to:
Table 3. Multipliers for various solar-cell areas according to the experimental design.

$$
n(x) n e^{-\left(x_{n}-x\right)^{2} / l^{2}}
$$

where $l_{D}$ is the Debye length, given by:

$$
l_{D}=\sqrt{\frac{K T \varepsilon}{q^{2} N_{D}}}
$$

$K$ is Boltzmann's constant, and T is the temperature.

\section{CALCULATION PARAMETERS FOR THE DERIVATION OF THE INTERNAL ELECTRIC FILED}

The impurity doping concentrations for a $p$ - $n$ junction process for solar cells are shown in Table 1. These are standard values of impurity doping parameters for epitaxial growth using a metal organic chemical-vapor developer process.

The doping impurity concentrations in solar-cell fabrication are determined by the different electrical properties in the two kinds of surface material. For $n$-type, the concentration is determined by $\mathrm{Si}_{2} \mathrm{H}_{6}$ in the IV compound, and for $p$-type, by DEZn in the II compound.

Table 2 shows an experimental design for solar-cell chargedensity calculations.

The design multiplier for different solar-cell areas of standard, type 1 , type 2 , type 3 , type 4 , and type 5 designs are shown in Table 3 . The solar-cell area is calculated as width $(\mathrm{cm}) \times$ length $(\mathrm{cm})$. The solar-cell area is directly related to the short circuit current (A). However, the relation between power-conversion efficiency $(\eta)$ and area is not a direct relationship.

\section{CONCLUSIONS}

In this section, the results obtained on the basis of the chargedensity fabrication experimental design will be discussed. The discovery of the $p$ - $n$ junction is usually attributed to American physicist Russell Ohl of Bell Laboratories [3]. Variations in the $p-n$ junction depletion-region parameters of a solar cell with impurity doping concentration are shown in Fig. 2. Parameter values are shown for both positive-type and negative-type transi- 


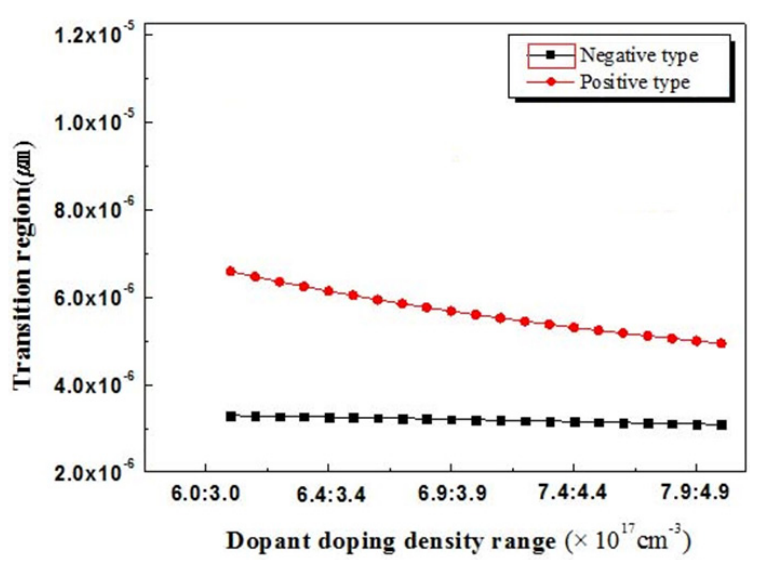

Fig. 3. Transition-region as a function of impurity doping concentrations.

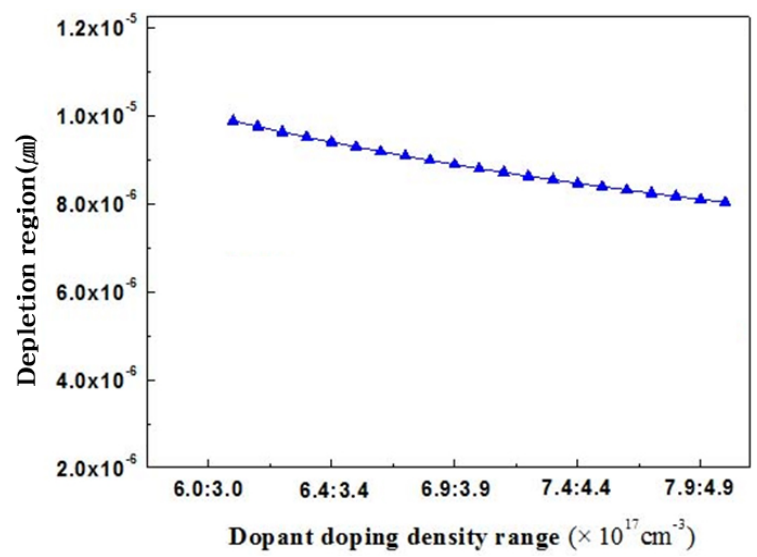

Fig. 4. Depletion-region as a function of Negative type and Positive type in amount.

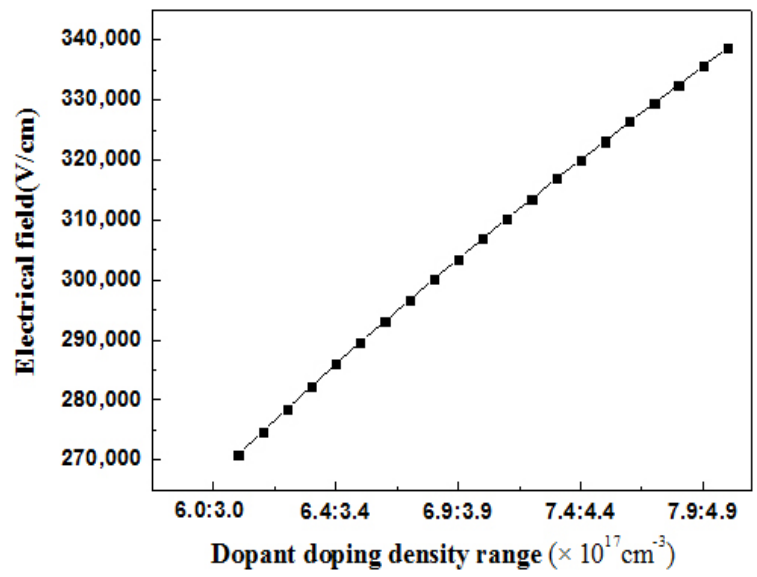

Fig. 5. Variation in electric field with impurity doping concentration in the $p-n$ junction of a solar cell.

tions. Both types of transition parameter depend on the impurity doping concentration. However, fabricated solar-cell area is unrelated to charge density over most of the depletion region.

The depletion parameter for the normal range of standard impurity doping concentrations was calculated as $8.0 \times 10^{-6} \mu \mathrm{m}$. In addition, the $p-n$ junction electric-field parameters of the solar cell were determined in relation to the ionization carrier, which

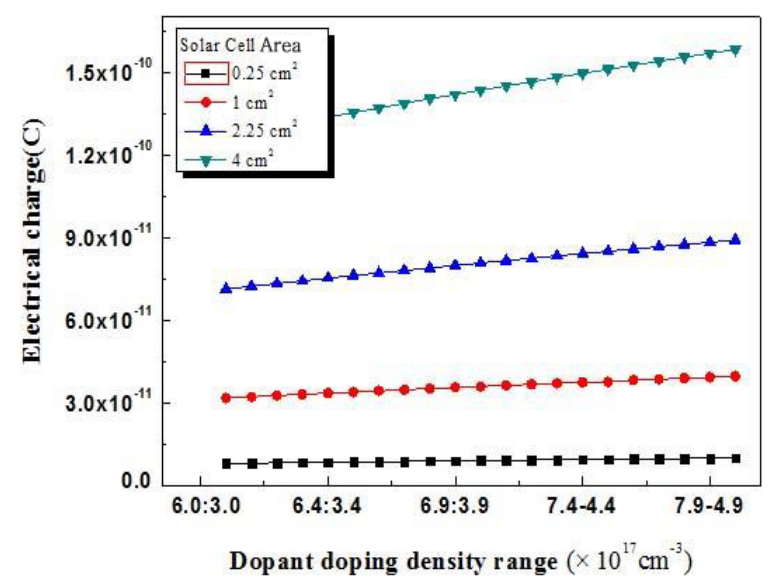

Fig. 6. Variations in electrical charge (C) with impurity doping concentration and solar-cell area for the type 1 data shown in Table 2.

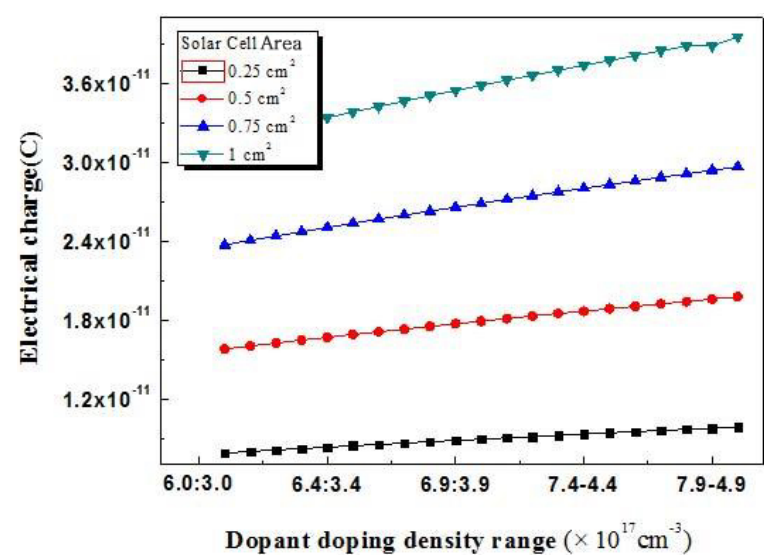

Fig. 7. Variations in electrical charge (C) with impurity doping concentration and solar-cell area for the type 2 data shown in Table 2 .

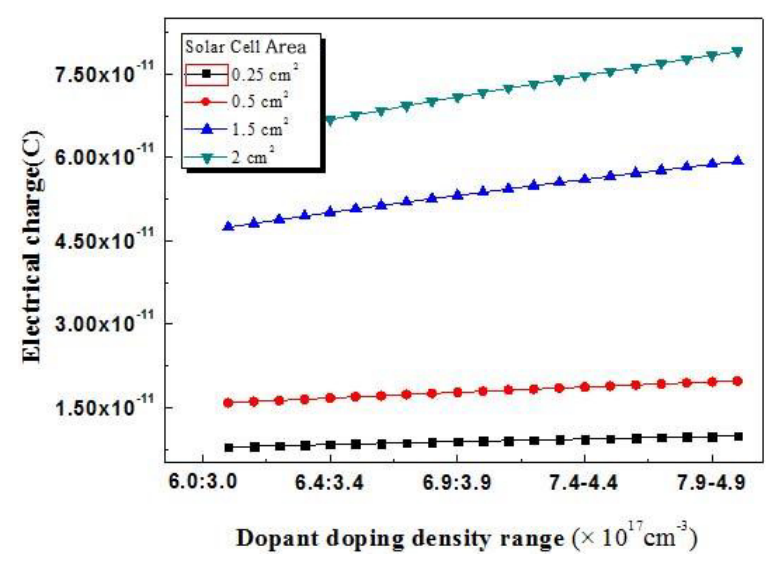

Fig. 8. Variations in electrical charge (C) with impurity doping concentration and solar-cell area for the type 3 data shown in Table 2.

includes both positive and negative ions. The minimum dopant impurity concentrations were $6.0 \times 10^{17} \mathrm{~cm}^{-3}$ and $3.0 \times 10^{17} \mathrm{~cm}^{-3}$ for $\mathrm{Si}_{2} \mathrm{H}_{6}$ and DEZn respectively. The maximum impurity doping concentrations were $8.0 \times 10^{17} \mathrm{~cm}^{-3}$ and $5.0 \times 10^{17} \mathrm{~cm}^{-3}$ for $\mathrm{Si}_{2} \mathrm{H}_{6}$ and DEZn respectively. Figure 5 shows the variation of the $p-n$ junction electric field with impurity doping concentration. In this plot, the depletion region is concentrated around the transi- 


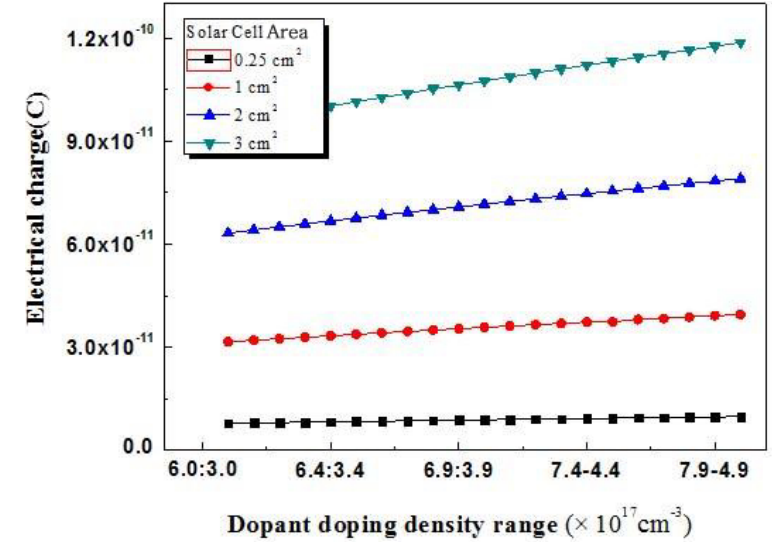

Fig. 9. Variations in electrical charge (C) with impurity doping concentration and solar-cell area for the type 4 data shown in Table 2 .

tion region and depends on impurity doping concentrations of both negative and positive types. The transition region is determined by the ionization carrier and by changes in the Fermi level due to variations in impurity doping concentrations.

The electric field created by the space charge region opposes the diffusion process for both electrons and holes. Figures 6-10 show changes in charge density which reflect the results obtained for the fabricated solar cells.

The fabricated solar-cell experimental design was implemented for standard, type 1 , type 2 , type 3 , type 4 , and type 5 solar cells. The charge density was found to be very high for the solar cell of $4.5 \mathrm{~cm}^{2}$ area. In addition, the charge density was found to increase with increasing solar-cell area. The carrier density was determined with silicon and GaAs material.

So far, this discussion has considered the basic p-n junction structure. The average length that an electron travels through the p-type material before recombining is called the diffusion length and is typically on the order of microns [4]. The built-in potential barrier maintains an equilibrium between the majority carrier electrons in the $n$ region and the minority carrier electrons in the $p$ region, and also between the majority carrier holes in the $p$ region and the minority carrier holes in the $n$ region [5]. Most semiconductor devices contain at least one junction between a $p$-type and an $n$-type semiconductor.

In conclusion, this research has examined variations in the charge density according to a solar-cell fabrication experimental

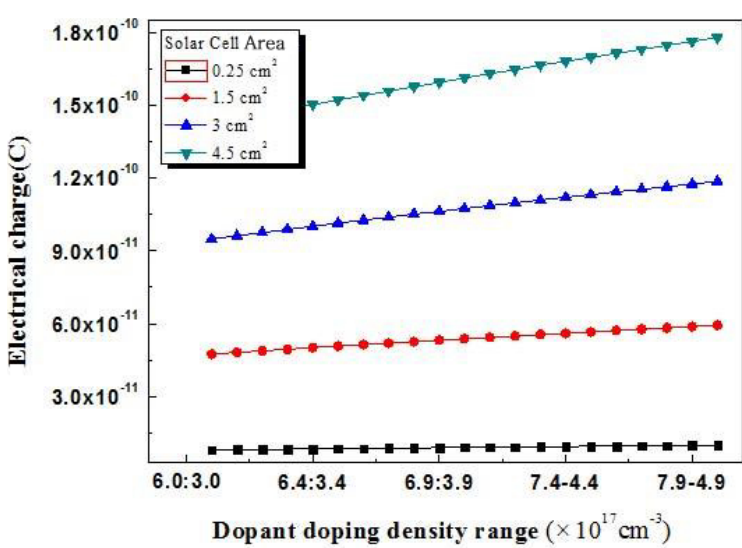

Fig. 10. Variations in electrical charge (C) with impurity doping concentration and solar-cell area for the type 5 data shown in Table 2.

design. The current was found to vary as a function of the charge density and the solar-cell area. The Fermi level was also affected by the impurity doping concentration; in the depletion region, the relationship was direct. The ionization carrier is a space of continuous motion in the osmosis interface between the two kinds of semiconductors. A mathematical foundation was also presented in this paper for the analysis of charge-density variations as a function of solar-cell area.

\section{REFERENCES}

[1] Korea Chemicals Management Association, The Compilation Organization of a Chemical Term Dictionary, The Direction of Chemical Term (Il Jin Publishing, Korea, 2006).

[2] V. Swaminathan and A. T. Macrander, Materials Aspects of GaAs and InP Based Structures (Prentice Hall, Englewood Cliffs, 1991) p. 242.

[3] W. W. Norton \& Company, Crystal Fire: The Birth of the Information Age, eds. M. Riordan and L. Hoddeson (Norton, New York, 1997)

[4] J. R. Hook and H. E. Hall, Solid State Physics, 2nd ed. (Wiley, New York,1995).

[5] D. A. Neamen, Semiconductor Physics and Devices: Basic Principles, 3rd ed. (McGraw-Hill, Boston, 2003). 